\title{
Synthesis of Novel Hexathiolated Squalene and Its Thiol-Ene Photopolymerization with Unsaturated Monomers
}

\author{
Ricardo Acosta Ortiz ${ }^{1 *}$, Ena Adeligna Obregón Blandón ${ }^{2}$, Ramiro Guerrero Santos ${ }^{1}$ \\ ${ }^{1}$ Centro de Investigación en Química Aplicada, Saltillo, México \\ ${ }^{2}$ Universidad Nacional de Ingeniería, Avenida Universitaria, Managua, Nicaragua \\ Email: ${ }^{*}$ racosta@ciqa.mx
}

Received February 3, 2012; revised March 6, 2012; accepted March 15, 2012

\begin{abstract}
In this work is described the synthesis of a multifunctional thiolated squalene. Thiol-ene coupling reactions were employed to functionalize the six double bonds of squalene, using thiolacetic acid. Hydrolysis of the resulting thioacetates, rendered the corresponding hexathiolated squalene SQ6SH. This compound was further photopolymerized separately with triallyl cyanurate, pentaerythritol triacrylate and diethyleneglycol divinyl ether. Real Time FTIR kinetics revealed that homopolymerization of the ene monomers took place in addition to the thiol-ene photopolymerization. Flexible films were obtained when SQ6SH was photopolymerized in bulk with the above mentioned unsaturated monomers.
\end{abstract}

Keywords: Squalene; Thiol-Ene; Photopolymerization; Biomaterials; Kinetics

\section{Introduction}

The search for new biobased raw materials to prepare polymers, has become an important issue due to increasing depletion of oil sources. A wide array of compounds like, terpenes [1,2], vegetable oils [3,4], cellulosic materials $[5,6]$, carbohydrates $[7,8]$, etc., have been used either as they are found naturally or chemically modified, to prepare polymeric materials.

Squalene is a triterpenoid found in shark liver, in wheat germ, and in olive oil. It is also present in the human body at $5 \%-8 \% \mathrm{w} / \mathrm{w}$ in the body fat [9]. It is produced industrially from the olive oil and it has found uses in the cosmetics industry and as skin humectant. Polyunsaturation of this compound make it a very attractive candidate to use it in the preparation of biobased polymers.

The "Click" thiol-ene Chemistry has acquired renewed interest due to the inherent characteristic of this kind of reactions. For instance, these reactions are very rapid, proceed almost quantitatively, and are not inhibited either by oxygen or humidity [10-12]. If monofunctional thiols and alkenes are irradiated with UV light, occurs thiol-ene coupling, but if difunctional or multifunctional thiols and alkenes are used, photopolymerization takes place.

In Scheme $\mathbf{1}$ is depicted the mechanism of thiol-ene photopolymerizations [13]. Initiation involves the photo-

${ }^{*}$ Corresponding author.

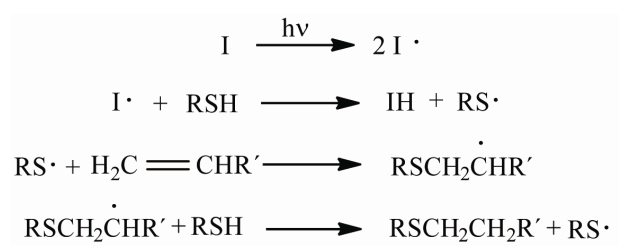

(1)

Scheme 1. Mechanism of thiol-ene photopolymerization.

lysis of the photoinitiator when the photocurable system is irradiated with UV light. Then the produced primary radicals abstract the hydrogen atom of thiol groups forming thiyl radicals. These radicals can react with the double bonds of the unsaturated compound forming a secondary radical which in turn can abstract the hydrogen atom of a second molecule of the thiol generating a new thiyl radical to repeat the cycle.

In continuing with our effort to utilize renewable starting materials to prepare monomers for thiol-ene phototopolymerization, the main objective of this research was to develop a methodology to prepare thiols using biobased multiunsaturated compounds, such as vitamin A, polyisoprene and squalene. This last compound was preferred to start with, because of its low molecular weight and ease of handling. The hexathiolated squalene was synthesized and subsequently photopolymerized with unsaturated monomers. Kinetics of photopolymerization was determined by means of Real-Time FTIR spectroscopy. 


\section{Experimental Section}

\subsection{Materials and Equipment}

Squalene (SQ), thiol acetic acid (TAA), sodium hydroxide, 2,2-Dimethoxy-2-phenylacetophenone (DMPA), diethylenglycol divinyl ether (DEGVE), triallyl cyanurate (TAC), pentaerythritol triacrylate (PETA), were all reagent grade and purchased from Aldrich Co (Toluca, Mexico). In Scheme 2 are shown the chemical structures of SQ, of the prepared compound 2, 6, 10, 15, 19, 23, hexamethyl tetracosane-3, 7, 11, 14, 18, 22-hexathiol (SQ6SH) and those of the unsaturated monomers mentioned above. Routine infrared spectra and photopolymerization kinetics were performed on a Magna Nicolet 550 Infrared spectrometer (Middleton, WI). NMR spectra were obtained using a 300 MHZ Jeol NMR spectrometer. Perkin Elmer Elemental Analyzer Series II CHNS/O 2400 (Waltham, MA).

\subsection{Synthesis of the Hexathioacetate of Squalene (SQ6TA)}

In quartz tube were weighed $1 \mathrm{~g}\left(2.43 \times 10^{-3} \mathrm{~mol}\right)$ of SQ, $2.22 \mathrm{~g}\left(2.92 \times 10^{-2} \mathrm{~mol}\right)$ of TAA and $1.25 \times 10^{-2} \mathrm{~g}(4.86$ $\times 10^{-5} \mathrm{~mol}$ ) of DMPA. Once that all components were added to the tube, it was placed in an UV light oven provided with a $300 \mathrm{~W}$ Fusion lamp. The tube was irradiated during 15 minutes and after this time the tube was allowed to cool to room temperature. The reaction mixture was dissolved in chloroform and extracted with $10 \%$ $\mathrm{NaOH} \mathrm{w} / \mathrm{w}(3 \times 30 \mathrm{~mL})$ in order to eliminate the excess of TAA. Then, the organic phase was washed with distilled water and subsequently dried with anhydrous sodium sulfate. The solvent was rotoevaporated and the crude SQ6TA was obtained at 85\% yield.

\subsection{Synthesis of SQ6SH}

In a three neck round-bottomed flask provided with condenser, thermometer, nitrogen inlet and magnetic stirring, were added $5.5 \mathrm{~g}\left(6.34 \times 10^{-3} \mathrm{~mol}\right)$ of the crude SQ6TA, $1.1 \mathrm{~g}\left(2.75 \times 10^{-2} \mathrm{~mol}\right)$ of powdered $\mathrm{NaOH}$ and $40 \mathrm{~mL}$ of methanol. Vigorous stirring was needed to dissolve the sodium hydroxide. Then, the reaction mixture was refluxed by 5.5 hours under nitrogen atmosphere. Afterwards, the solvent of reaction mixture was evaporated and the residue was dissolved in water and acidified with a $0.1 \mathrm{~N}$ HCL solution. The aqueous phase was extracted with chloroform, $(3 \times 30 \mathrm{~mL})$ and the resulting organic phase was washed with distilled water, dried with anhydrous sodium sulfate and rotoevaporated to eliminate the solvent. The crude product was further purified by column chromatography using silica gel as support and a mixture hexane: ethyl acetate 98:2 as eluent. A yellowish liquid with astringent odor was obtained at $77 \%$ yield. Elemental Analysis: Theory C 58.57, H 10.16, S 31.27, Experimental C 58.22, H 9.96, S 31.06.

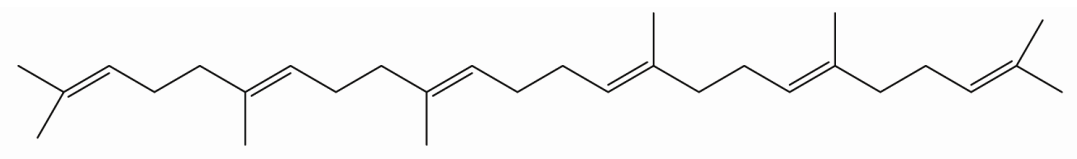

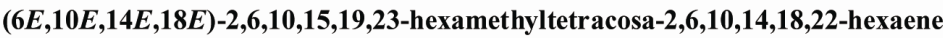
SQ<smiles>CC(C)C([SiH3])CCC(C)C(S)CCC(C)C(S)CCC(S)C(C)CCC(S)C(C)CCC(S)C(C)C</smiles>

2,6,10,15,19,23-hexamethyltetracosane-3,7,11,14,18,22-hexathiol SQ6SH
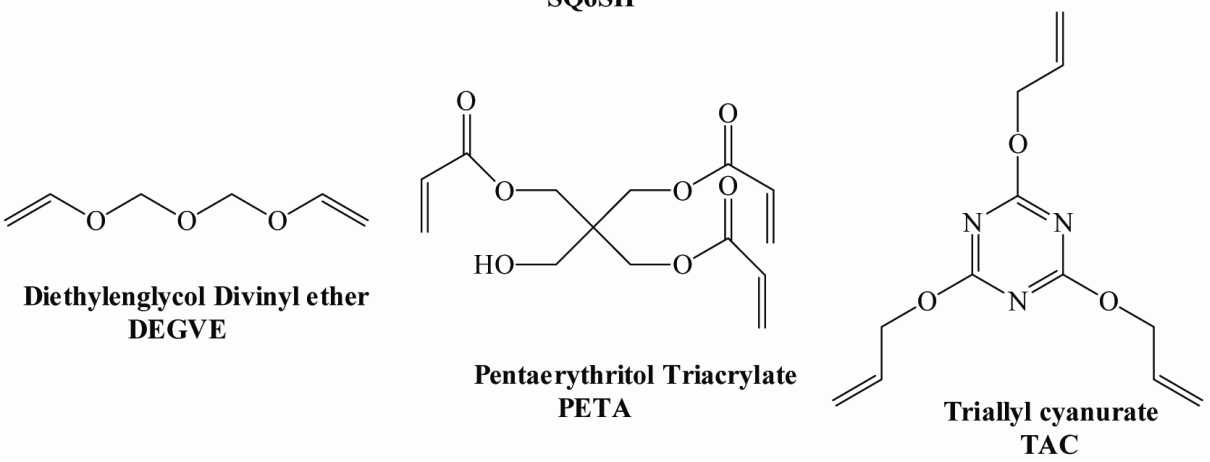

Scheme 2. Chemical structures of squalene (SQ), of synthesized compound SQ6SH and of ene monomers used in this study. 


\subsection{Determination of Kinetics of Thiol-Ene Photopolymerizations by RT-FTIR}

RT-FTIR was used to monitor the kinetics of photopolymerization of the synthesized monomer SQ6SH with a Nicolet Magna 550 FT-IR spectrometer equipped with a DTGS detector fitted with a UVEXS model SCU 110 mercury lamp. The intensity of the UV irradiation was measured with a UV Process Supply Inc. Control cure radiometer. Thiol-ene formulations were prepared by mixing $0.5 \mathrm{~g}$ of SQ6SH with each of the ene monomers in stoichiometric ratios, considering the number of functional groups. DMPA was added to each formulation at 1 mol \%. All kinetics experiments were conducted at $25^{\circ} \mathrm{C}$. The course of the photopolymerization was followed by simultaneously monitoring the decrease of the peaks of the corresponding functional groups. For instance, the thiol was monitored following the infrared absorption band at $2560 \mathrm{~cm}^{-1}$ due to its S-H group. Allyl group conversion was monitored using the carbon-carbon double bond absorption peak at $1646 \mathrm{~cm}^{-1}$. Each kinetic run was carried out a minimum of five times. Data were collected at a rate of one spectrum per second and processed with the OMNIC Series software. Conversions were calculated using the ratio of peak areas to the peak area prior to photopolymerization. The kinetic parameter $\mathrm{R}_{\mathrm{p}} / \mathrm{M}_{0}$, was determined from the initial slopes of the irradiation time-conversion curves according to Equation (5):

$$
\mathrm{R}_{\mathrm{p}} / \mathrm{M}_{0}=([\text { conversion }] \mathrm{t} 2-[\text { conversion }] \mathrm{t} 1) /(\mathrm{t} 2-\mathrm{t} 1)
$$

where $R_{p}$ and $M_{0}$ are respectively the rate of photopolymerization and the initial monomer concentration as well as the conversions are as determined from the curves at irradiation times $\mathrm{t} 1$ and $\mathrm{t} 2$. The obtained profile of diminution of the absorbance of a determined peak, using the Series software included in OMNIC, is processed in excel, by using the Equation (6), where $\mathrm{Ct}$ is the conversion at any time, $\mathrm{B}_{0}$ is the absorbance of the peak at zero time and $\mathrm{Bt}$ is the absorbance at any given time

$$
\mathrm{Ct}=\left[\mathrm{B}_{0}-\mathrm{Bt} / \mathrm{B}_{0}\right] \times 100
$$

In Table $\mathbf{1}$ are shown the amounts and ratios of the comonomers used in the determination of the photopoly-

Table 1. Amounts and ratios of SQ6SH and of the three ene monomers. SQ6SH was formulated separately with each of the three monomers and $3 \mathrm{~mol} \%$ of DMPA.

\begin{tabular}{cccccc}
\hline & SQ6SH & DEGVE & TAC & PETA & DMPA \\
\hline Equation & 1 & 3 & 2 & 3 & 0.03 \\
mol & $3.25 \mathrm{E}-04$ & $9.75 \mathrm{E}-04$ & $6.50 \mathrm{E}-04$ & $6.50 \mathrm{E}-04$ & $9.75 \mathrm{E}-06$ \\
$\mathrm{MW}(\mathrm{g} / \mathrm{mol})$ & 615.2 & 158.2 & 249.27 & 298.30 & 256.30 \\
$\mathrm{~g}$ & 0.2 & 0.15 & 0.16 & 0.19 & 0.002 \\
\hline
\end{tabular}

merization kinetics by RT-FTIR.

\subsection{Study by DSC}

The Tg's of the prepared polymers were measured on DSC2920 thermal analyzer (TA instruments, Inc.) with a heating rate of $10^{\circ} \mathrm{C} / \mathrm{min}$ under nitrogen atmosphere. The average weight of test samples was 3 - $5 \mathrm{mg}$.

\section{Results and Discussion}

\subsection{Synthesis of SQ6SH}

The development of monomers based on renewable source derived materials has been one of the main interests of our research group. Previously, we have reported the use of vegetable oil [14], sucrose [15-17] and isosorbide [18], as starting materials to prepare epoxy monomers for cationic photopolymerization, and allylated carbohydrates to use them as comonomers in thiol-ene photopolymerization. To go further to obtain purely biobased polymers using the thiol-ene photopolymerization technique, we needed to prepare multifunctional thiols derived from natural sources. The "Click" thiol-ene coupling between an unsaturated compound such as SQ with TAA under UV irradiation, and subsequent hydrolyzation of the obtained thioacetate, proved to be an effective method to synthesize the hexafunctional thiol (see Scheme 3). Here, is worth mentioning that hydrothiolation of internal double bonds is not as fast as that of terminal double bonds. Hoyle, et al., [19,20] found that hydrothiolation of 1-hexene is eight times faster than that of trans- 2-hexene and 18 times faster than of trans-3-hexene. This difference in reactivity was attributed to steric effects and also to the reversibility of the addition reaction of the thyil radical to the double bond. As in our case, all double bonds of SQ are internal, adjacent to a methyl group and of type trans, they were not very reactive, therefore, it was necessary to add a $100 \%$ excess of TAA to force the completion of the reaction. Figure 1 shows the ${ }^{1} \mathrm{H}$ NMR spectrum of SQ6TA. It can be observed the presence of the peak at $2.3 \mathrm{ppm}$ corresponding to the methyl of the thioacetate groups and the absence of the signals at 5 ppm corresponding to the double bonds of SQ. The proton NMR spectrum of SQ6SH depicted in Figure 2 reveals the disappearing of the peak at $2.3 \mathrm{ppm}$. Also, in Figure 3 are shown the IR spectra of the starting material SQ, of the intermediate compound SQ6TA and that of the final product where the SH peak is marked at 2560 $\mathrm{cm}^{-1}$.

\subsection{Photopolymerization Studies}

The prepared SQ6SH was photopolymerized with three types of unsaturated monomers, namely, vinyl ether (DEGVE), allyl isocyanurate (TAC) and pentaerithritol 


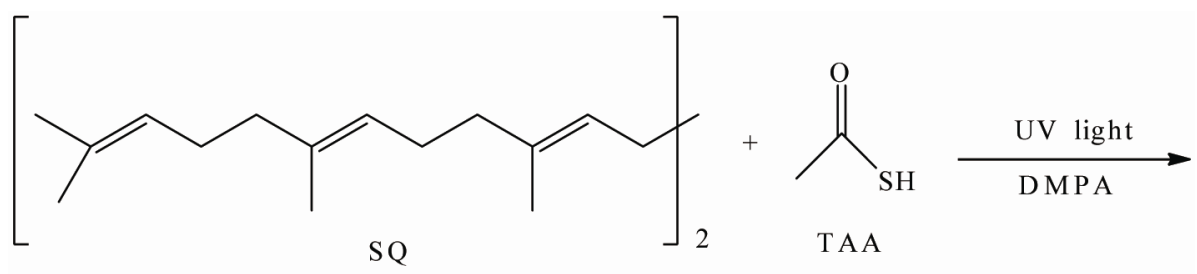<smiles>CC(=O)SC(CCC(C)C(CCC(C)C(CC(C)(C)C)SC(C)=O)SC(C)=O)C(C)C</smiles>

SQ6T A<smiles>CC(C)C(S)CCC(C)C(S)CCC(C)C(S)CC(C)(C)C</smiles>

SQ6SH

Scheme 3. Methodology to prepare SQ6SH.

triacrylate (PETA). All three compounds were selected based on their different reactivities. The reactivity depends on the substitution in the double bond group. The higher the electron density in the double bonds the higher the reactivity. Figure 4 shows the kinetics curves obtained by RT-FTIR of the photopolymerization of the photocurable system SQ6SH/TAC. It was observed that this photocurable system was rather reactive, with the double bonds groups being consumed more rapidly and with higher conversion than the thiol groups. As in the step-growth polymerization, both thiol and ene compounds must be consumed at the same time, it is clear that two polymerization mechanisms are occurring concurrently: the thiol-ene photopolymerization and the radical homopolymerization of TAC. On one hand, although it has been reported that allylic bonds can homopolymerize to some extent [21], in our case, monomer TAC polymerize readily achieving $42 \%$ conversion in the first 20 seconds and $75 \%$ after 120 seconds. On the other hand, low conversion of thiol group (25\%) was produced in the same run as a result of the multifuncionality of SQ6SH. As usual, in thiol-ene photopolymerizations, if multifunctional enes and thiols are used, crosslinked polymers are obtained. The higher the multifunctionality, the higher the crosslink density in the produced polythioether. Lower conversions of comonomers are achieved as a result of the higher amount of trapped functional groups in the tridimensional crosslinked network. One of the main characteristics of the thiol-ene photopolymerization is the delayed onset of gel formation in comparison with the radical photopolymerization of acrylates. Jacobine and collaborators [22] proposed an equation (Equation (7)) to calculate the gel point of thiol-ene systems considering the functionality of comonomers:

$$
\alpha=\sqrt{\frac{1}{r\left(f_{\text {thiol }}-1\right)\left(f_{\text {ene }}-1\right)}}
$$

where $\alpha$ is the gel point, $r$ is the thiol-ene molar ratio based on functional groups, $f_{\text {thiol }}$ is the thiol functionality and $f_{\text {ene }}$ is the ene functionality. In the case of our photocurable system TAC/SQ6SH, as the functionality of thiol compound is 6 and that of the ene compound is 3 , the gel point obtained by using Equation (1), is 22\%, which corresponds fairly with the observed conversion of SQ6SH. The difference between the conversion of the thiol and that of the ene compound correspond to the amount of homopolymerization of TAC.

Figure 5 depicts the kinetics curves of double bonds of DEGVE and of thiol groups of SQ6SH. It was observed 


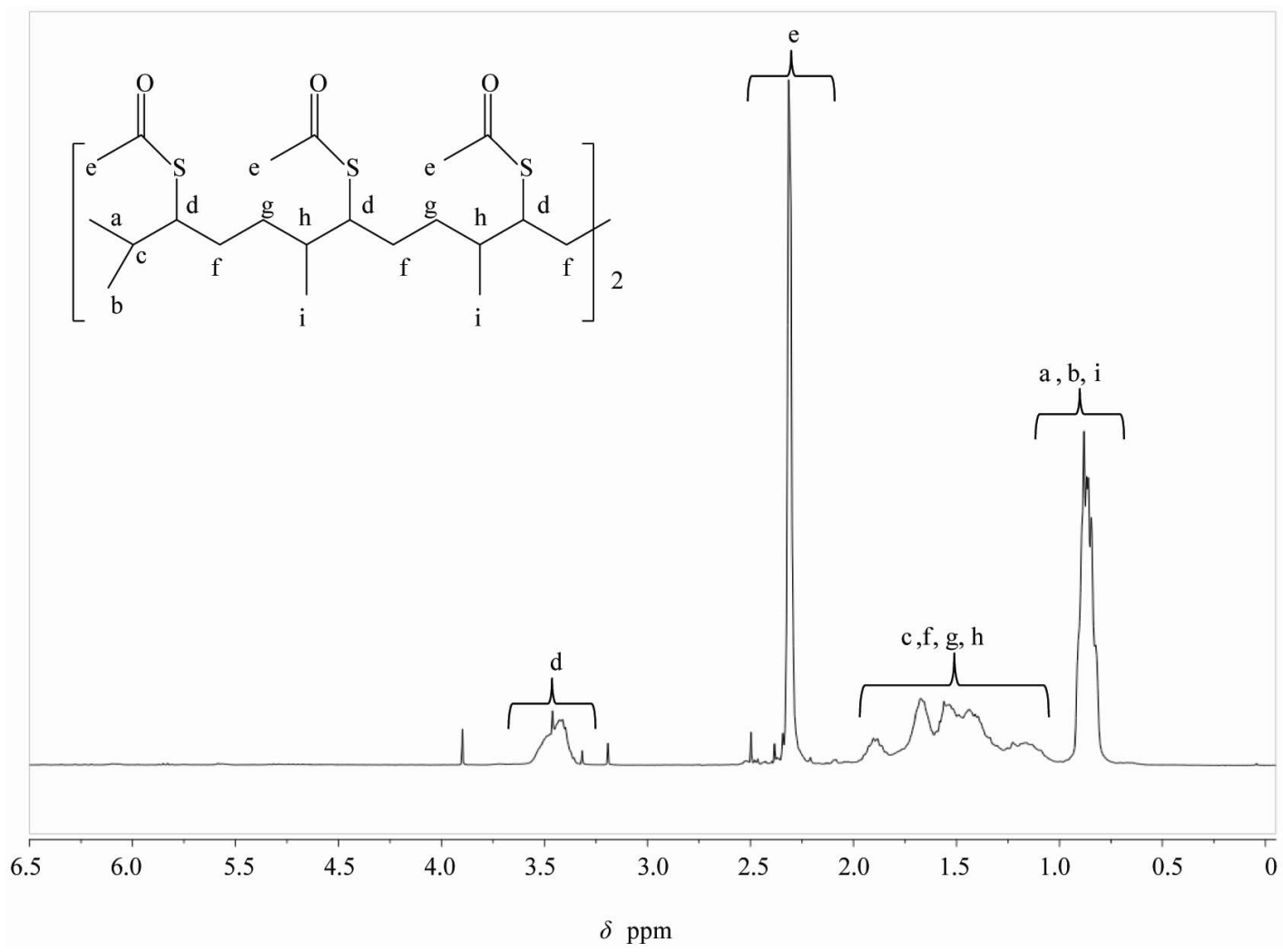

Figure 1. ${ }^{1} \mathrm{H}$ NMR spectrum of SQ6TA run in $\mathrm{CDCl}_{3}$.

a similar situation than in the case of TAC, as in this case it also took place homopolymerization of vinyl ether in addition to the step-growth thiol-ene photopolymerization, although it was slightly slower than the formulation with TAC. The hexafunctionality of SQ6SH and the bifunctionality of DEGVE resulted in 25\% conversion of the thiol groups (theory 25\%).

The kinetics curves of the system PETA/SQ6SH are shown in Figure 6. Although PETA is also trifunctional, the achieved conversion for the thiol groups was $17.5 \%$. This apparently slightly lower conversion could be explained by the higher reactivity of PETA, as the homopolymerization of this monomer was somewhat faster than the other two ene monomers. In this way, the chances to react with the thiol are reduced to some extent.

\subsection{DSC of Produced Films}

The formulations of SQ6SH with the three unsaturated monomers were photopolymerized in bulk, as thin films on glass slides. Flexible films were obtained with subzero Tg's. The values of Tg of the obtained polymers are shown in Table 2. In the resulting mixture of polymers, the inherent high mobility of the aliphatic hexathiolated squalene backbone added to the flexibility imparted by the tioether groups resulted in the formation of polymers
Table 2. Tg's of the polymers derived from SQ6SH with the three ene monomers and from their homopolymers. The Tg was determined by DSC considering the second heating of the sample.

\begin{tabular}{lc}
\hline \multicolumn{1}{c}{ Sample } & $\mathbf{T g}^{\circ} \mathbf{C}$ \\
\hline SQ6SH/TAC & -22.61 \\
SQ6SH/PETA & -25.12 \\
SQ6SH/DEGVE & -20.29 \\
TAC & 28.75 \\
PETA & 25.24 \\
DEGVE & 41.02 \\
\hline
\end{tabular}

with Tg's around $-20^{\circ} \mathrm{C}$. For comparison pristine Ene monomers were also polymerized in bulk and the Tg of these polymers was also determined. It can be seen that the $\mathrm{Tg}$ of the photopolymers are around $50^{\circ} \mathrm{C}-60^{\circ} \mathrm{C}$ higher than that of the polymers obtained using the SQ6SH, due to the already mentioned higher flexibility of the formed polythioethers.

\section{Conclusion}

A novel biobased multifunctional thiolated comonomer was prepared using SQ as starting material. The comonomer was produced in two stages. First, SQ was irradiated in the presence of TAA to produce the hexathio- 


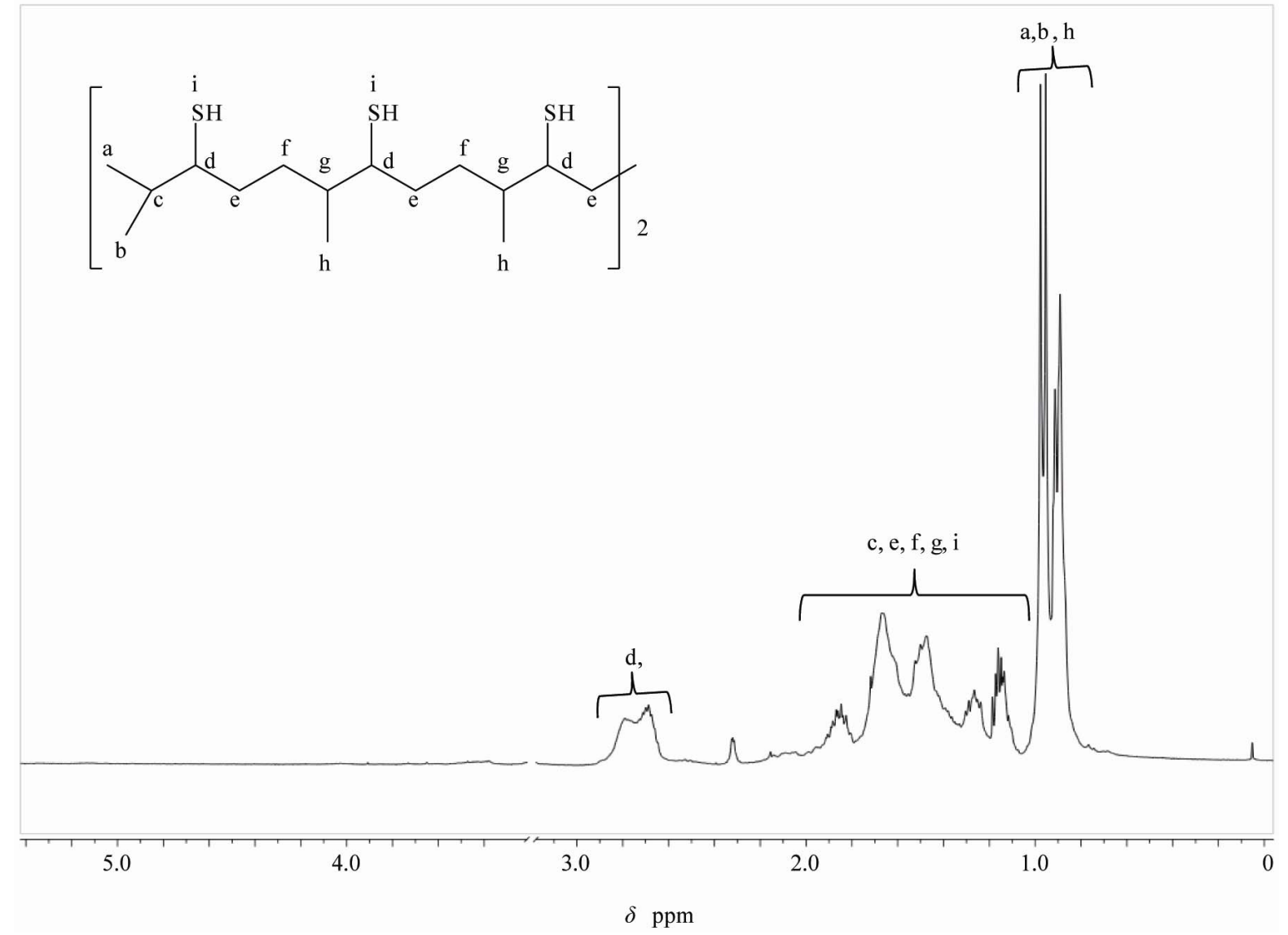

Figure 2. ${ }^{1} \mathrm{H}$ NMR spectrum of SQ6SH run in $\mathrm{CDCl}_{3}$.

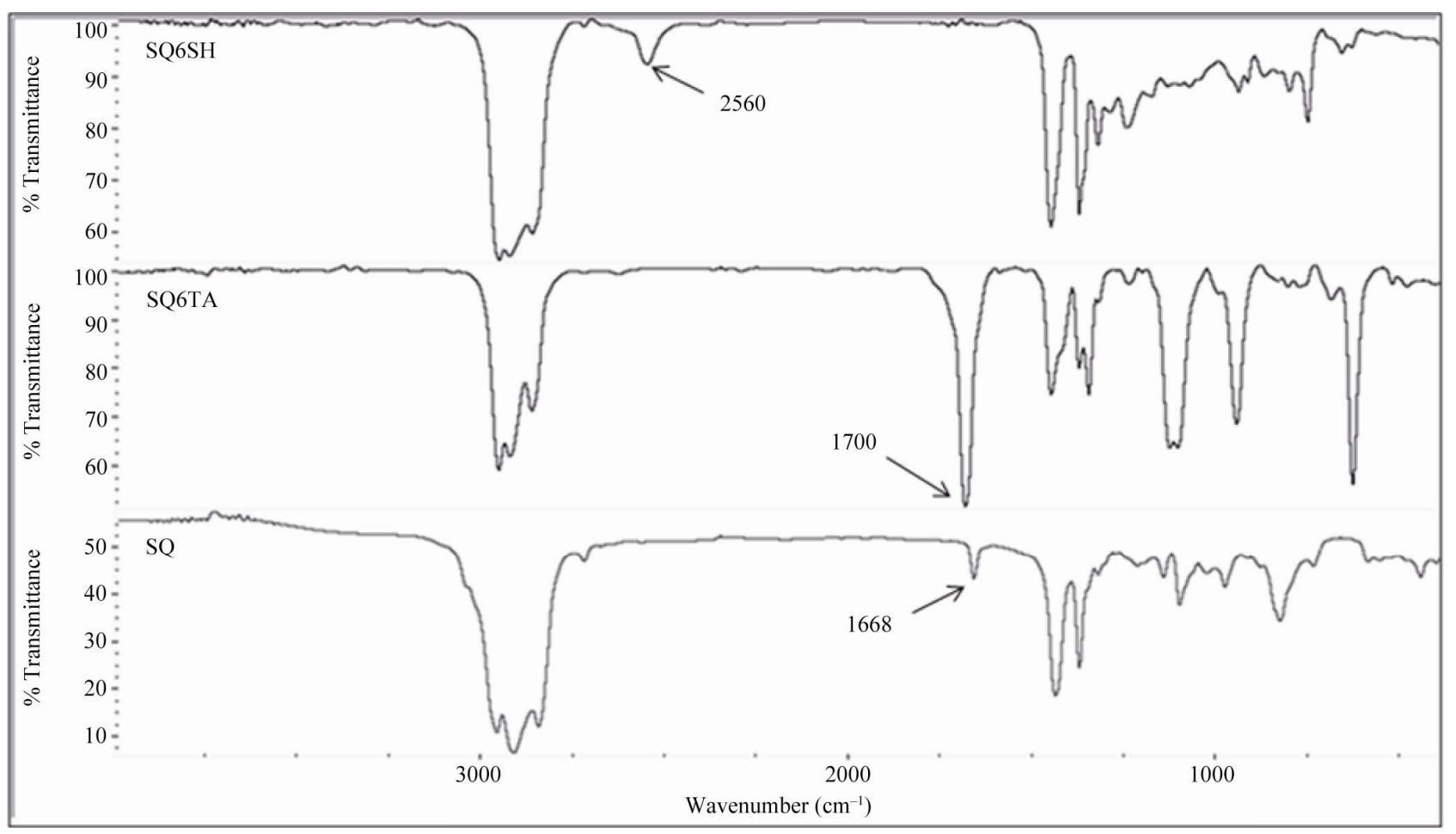

Figure 3. Comparison of FTIR spectra of SQ, SQ6TA and SQ6SH. 


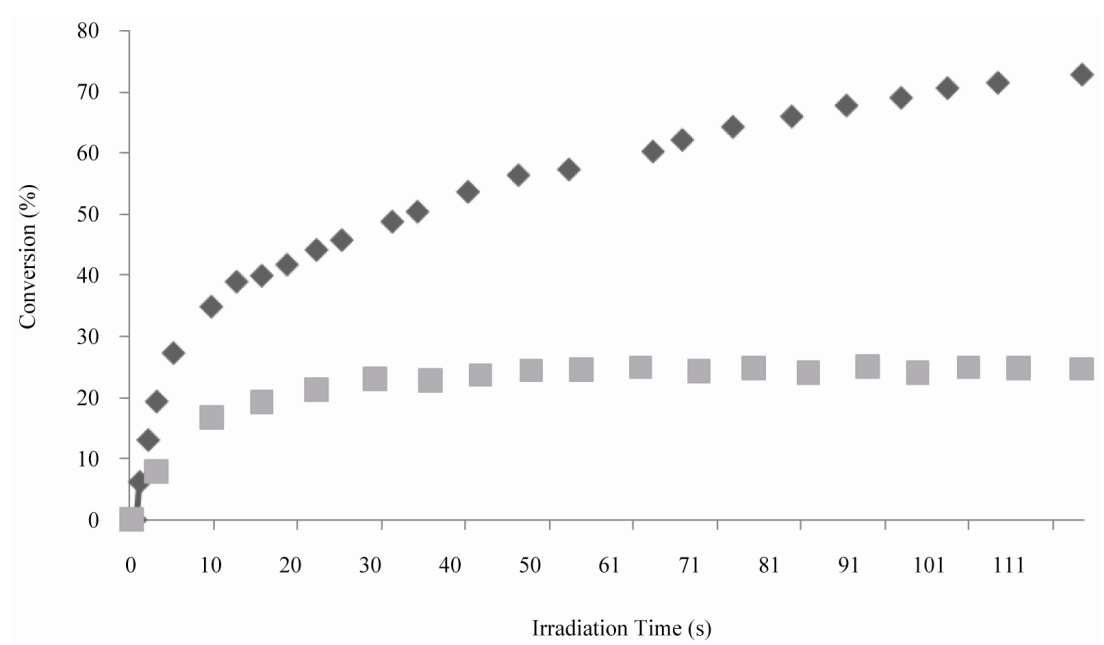

Figure 4. Comparison of conversion vs time curves for thiol groups of SQ6SH ( $\square$ ) and double bonds of TAC, ( $\diamond)$, UV light intensity was $15 \mathrm{~mW} / \mathrm{cm}^{2}$.

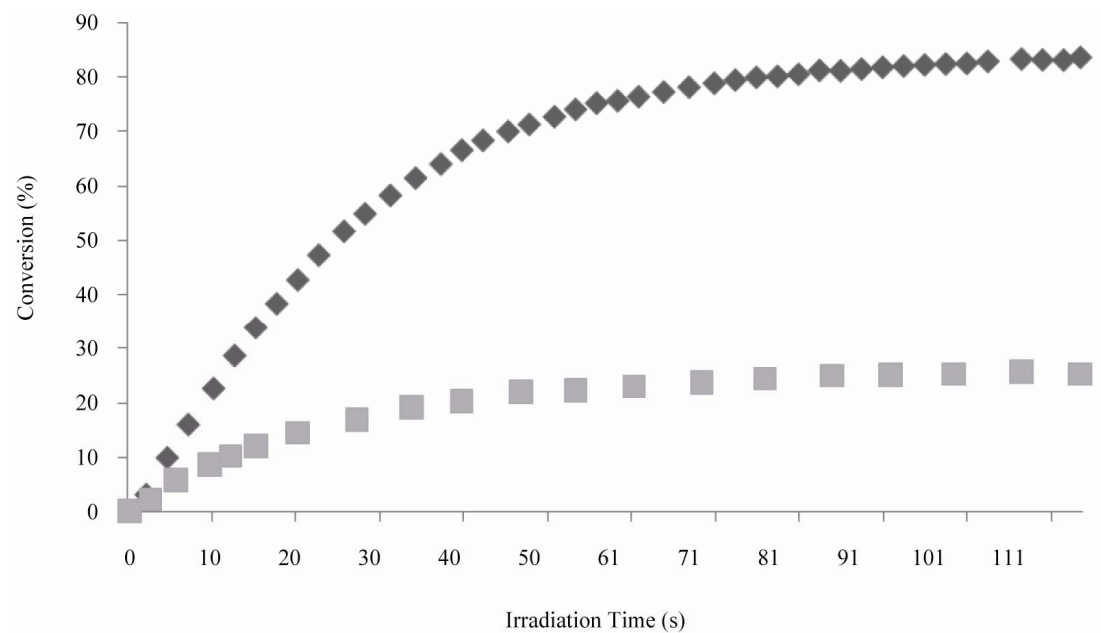

Figure 5. Comparison of conversion vs time curves for thiol groups of SQ6SH ( $\square$ ) and double bonds of DEGVE ( $\diamond)$, UV light intensity was $15 \mathrm{~mW} / \mathrm{cm}^{2}$.

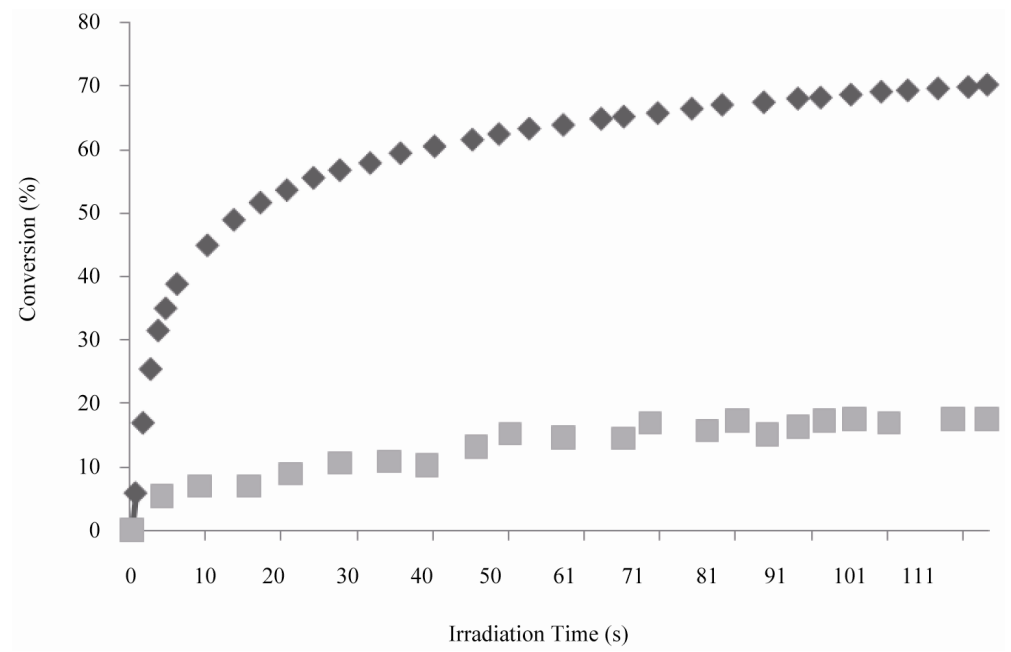

Figure 6. Comparison of Conversion vs Time curves for thiol groups of SQ6SH ( $\square$ ) and double bonds of PETA, ( $\bullet)$, UV light intensity was $15 \mathrm{~mW} / \mathrm{cm}^{2}$. 
ester. Then in the second stage the thioester was hydrolyzed in basic methanolic solution to obtain the hexathiolated monomer SQ6SH. Photopolymerization of this compound separately with three unsaturated monomer resulted in the formation of very flexible polythioether films with $\mathrm{Tg}$ 's in the range $-20^{\circ} \mathrm{C}$ to $-25^{\circ} \mathrm{C}$. The photocurable systems involving the prepared multifunctional thiol were rather reactive observing in all cases, that both homopolymerization and step-growth thiol-ene photopolymerization occurred concurrently.

\section{Acknowledgements}

The authors would like to thank the Mexican National Council of Science and Technology (CONACYT) for funding this project (151489). Assistance in analysis of the samples by Judith Cabello, Guadalupe Mendez and Julieta Sanchez is gratefully acknowledged.

\section{REFERENCES}

[1] O. Tueruenc, L. Montero de Espinosa, M. Firdaus and M. A. R. Meier, "Clicking Renewable Resources: Thiol-Ene Additions for the Synthesis of Monomers and Polymers Derived from Plant Oils and Terpenes," Polymer Preprints (American Chemical Society, Division of Polymer Chemistry), Vol. 51, No. 2, 2010, pp. 724-725.

[2] A. Gandini, "Polymers from Renewable Resources: A Challenge for the Future of Macromolecular Materials," Macromolecules, Vol. 41, No. 24, 2008, pp. 9491-9504. doi:10.1021/ma801735u

[3] H. G. Cho, S. Y. Park, J. Jegal, B. K. Song and H. J. Kim, "Preparation and Characterization of Acrylic Polymers Based on a Novel Acrylic Monomer Produced from Vegetable Oil," Journal of Applied Polymer Science, Vol. 116, No. 2, 2010, pp. 736-742.

[4] J. V. Crivello, "Biomass as a Source of Photopolymerizable Monomers and Polymers,” Polymer Preprints (American Chemical Society, Division of Polymer Chemistry), Vol. 48, No. 2, 2007, pp. 844-845.

[5] M. Rose and R. Palkovits, "Cellulose-Based Sustainable Polymers: State of the Art and Future Trends," Macromolecular Rapid Communications, Vol. 32, No. 17, 2011, pp. 1299-1311. doi:10.1002/marc.201100230

[6] B. B.-A. Bar-Nir and J. F. Kadla, "Synthesis and Structural Characterization of 3-O-Ethylene Glycol Functionalized Cellulose Derivatives," Carbohydrate Polymers, Vol. 76, No. 1, 2009, pp. 60-67. doi:10.1016/j.carbpol.2008.09.026

[7] T. Satoh, "High-Performance Carbohydrate Polymer Synthesized from Plant Source,” Bio-Industry, Vol. 26, No. 8, 2009, pp. 50-57.

[8] K. M. Mostafa, A. R. Samerkandy and A. A. El-Sanabay, "Modification of Carbohydrate Polymers. Part 2: Grafting of Methacrylamide onto Pregelled Starch Using Vanadium-Mercaptosuccinic Acid Redox Pair," Journal of Applied Sciences Research, Vol. 3, No. 8, 2007, pp. 681-
689.

[9] http://www.sophim.com/htmles/fsqualene.html

[10] A. B. Lowe, “Thiol-Ene 'Click' Reactions and Recent Applications in Polymer and Materials Synthesis," Polymer Chemistry, Vol. 1, No. 1, 2010, pp. 17-36. doi:10.1039/b9py00216b

[11] C. E. Hoyle and C. N. Bowman, "Thiol-Ene Click Chemistry," Angewandte Chemie International Edition, Vol. 49, No. 9, 2010, pp. 1540-1573. doi:10.1002/anie.200903924

[12] M. J. Kade, D. J. Burke and C. J. Hawker, "The Power of Thiol-Ene Chemistry," Journal of Polymer Science Part A: Polymer Chemistry, Vol. 48, No. 4, 2010, pp. 743-750. doi:10.1002/pola.23824

[13] A. F. Jacobine, “Polymerization Mechanisms,” In: J. D. Fouassier and J. F. Rabek, Eds., Radiation Curing in Polymer Science and Technology III, Elsevier, London, 1993.

[14] R. A. Ortiz, D. P. López, M. L. G. Cisneros, J. C. R. Valverde and J. V. Crivello, "A Kinetic Study of the Acceleration Effect of Substituted Benzyl Alcohols on the Cationic Photopolymerization Rate of Epoxidized Natural Oils,” Polymer, Vol. 46, No. 5, 2005, pp. 1535-1541. doi:10.1016/j.polymer.2004.12.020

[15] R. A. Ortiz, A. E. G. Valdez, M. G. M. Aguilar and M. L. B. Duarte, "An Effective Method to Prepare Sucrose Polymers by Thiol-Ene Photopolymerization," Carbohydrate Polymers, Vol. 78, No. 2, 2009, pp. 282-286. doi:10.1016/j.carbpol.2009.03.045

[16] R. A. Ortiz, Y. A. R. Martínez, A. E. G. Valdez and M. L. B. Duarte, "Preparation of a Crosslinked Sucrose Polymer by Thiol-Ene Photopolymerization Using Dithiotreitol as Comonomer," Carbohydrate Polymers, Vol. 82, No. 15, 2010, pp. 822-828. doi:10.1016/j.carbpol.2010.05.054

[17] A. E. G. Valdez, R. A. Ortiz, M. G. M. and M. L. B. Duarte, "Effect of the Degree of Substitution of the Diallyl Sucrose on the Reactivity and Physical and Mechanical Properties of the Polymers Obtained by the ThiolEne Photopolymerization Mechanism," Current Trends in Polymer Science, Vol. 13, 2009, pp. 25-36.

[18] R. A. Ortiz, Y. A. R. Martinez and A. E. G. Valdez, "Preparation of Biobased Polymers Derived from Isosorbide by Means of Thiol-Ene Photopolymerization,” Journal of Biobased Materials and Bioenergy, Vol. 6, No. 1, 2012, pp. 36-41.

[19] C. E. Hoyle, T. Y. Lee and T. Roper, “Thiol-Enes: Chemistry of the Past with Promise for the Future,” Journal of Polymer Science Part A: Polymer Chemistry, Vol. 42, No. 21, 2004, pp. 5301-5338. doi:10.1002/pola.20366

[20] T. M. Roper, C. A. Guymon, E. S. Jonsson and C. E. Hoyle, "Influence of the Alkene Structure on the Mechanism and Kinetics of Thiol-Alkene Photopolymerizations with Real-Time Infrared Spectroscopy," Journal of Polymer Science Part A: Polymer Chemistry, Vol. 42, No. 24, 2004, pp. 6283-6298. doi:10.1002/pola.20452

[21] N. B. Cramer and C. N. Bowman, "Kinetics of Thiol-Ene and Thiol-Acrylate Photopolymerizations with Real-Time Fourier Transform Infrared Spectroscopy,” Journal of Polymer Science Part A: Polymer Chemistry, Vol. 39, No. 
19, 2001, pp. 3311-3319. doi:10.1002/pola.1314

[22] A. F. Jacobine, D. M. Glaser, P. J. Grabek, D. Mancini, M. Masterson, S. T. Nakos, M. A. Rakas and J. G. Woods, "Photocrosslinked Norbornene-Thiol Copolymers: Syn- thesis, Mechanical Properties, and Cure Studies,” Journal of Applied Polymer Science, Vol. 45, No. 3, 1992, pp. 471-483. doi:10.1002/app.1992.070450312 